\title{
Microparticles and endothelial function-A tour de force
}

\author{
Victor A. Ferraris, MD, PhD
}

See related article on pages 666-72.

Circulating microparticles (MPs) are small, membranederived vesicles that come from activated or apoptotic cells (platelets, white cells, endothelium, tumor cells, and red blood cells). They lack a nucleus, but contain a lipid membrane, and are defined by their surface antigens, which reflect their cell of origin. Their size ranges from 50 to $1000 \mathrm{~nm}$ and should be distinguished from other groups of cell-derived vesicles, such as exosomes and apoptotic bodies. Exosomes are small vesicles $(40-100 \mathrm{~nm})$ that form through exocytosis, whereas MPs form mostly by reverse budding and fission of the plasma membrane. Because exosomes and MPs are often released concomitantly, differentiation of these 2 microvesicular species is difficult.

\section{WHY MICROPARTICLES?}

What makes MPs so interesting is their wealth of potential actions. They serve as biomarkers, procoagulant agents, participants in atherogenesis, agents of transfer of biologic information, cancer promoters, and sources of vascular dysfunction. ${ }^{1,2}$ Some examples of MP actions are described here.

First, MPs derived from tumor cells are involved in the transport of cancer proteins and in delivering micro ribonucleic acid to the surrounding healthy tissue. This process leads to a change of healthy cell phenotype and creates a tumor-friendly environment. Microparticles play an important role in tumor angiogenesis and in the degradation of matrix, owing to the presence of metalloproteases, which facilitate metastasis. ${ }^{3}$ Further, circulating endothelial-derived MPs may serve as useful biomarkers predictive of 1-year mortality in patients with advanced lung cancer.

Second, support with left ventricular assist devices leads to significant improvements in microvascular perfusion and hemodynamics, but at the expense of

\footnotetext{
From the Department of Surgery, University of Kentucky, Lexington, Ky.

Disclosures: Author has nothing to disclose with regard to commercial support.

Received for publication June 3, 2015; accepted for publication June 4, 2015; available ahead of print July 7, 2015.

Address for reprints: Victor A. Ferraris, MD, PhD, Department of Surgery, University of Kentucky, A301 Kentucky Clinic, 740 S Limestone, Lexington, KY 40536-0284 (E-mail: Ferraris@earthlink.net).

J Thorac Cardiovasc Surg 2015;150:673-4

0022-5223/\$36.00

Copyright (c) 2015 by The American Association for Thoracic Surgery

http://dx.doi.org/10.1016/j.jtcvs.2015.06.011
}

destruction of blood cells and creation of blood celland endothelial cell-derived MPs that contribute to residual endothelial dysfunction, possibly by increasing nitric oxide scavenging capacity.,

Third, platelet-derived MPs may play an important role in trauma-induced coagulop-

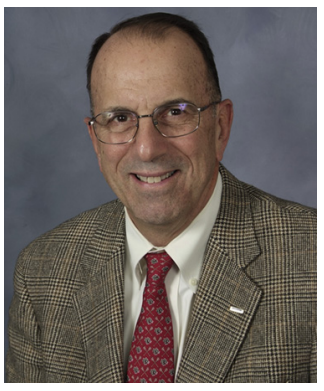
athy. ${ }^{7}$ In this setting, MPs may be important in clot formation in trauma patients, and increased numbers of red blood cell transfusions translate into low levels of platelet-derived MPs.

A fourth action is that drug encapsulation by MPs can prolong the effects of vasoactive drugs and increase drug concentrations in certain tissues. ${ }^{8,9}$ Encapsulation of milrinone within MPs provides a new strategy for drug delivery in patients who have end-stage heart failure. ${ }^{9}$ Finally, MPs play an important role in promoting endothelial stability and may serve as biomarkers of endothelial-related disease states. ${ }^{10}$

\section{THE AMBIVALENCE OF MICROPARTICLES}

Reports suggest that MPs have an ambivalent and diverse set of actions related to the vascular endothelium. ${ }^{11}$ The protein composition of MPs depends on the stimulus triggering their release from parent cells. Proteins on the surface of MPs originate mostly from the plasma membrane. Microparticles contain nuclear material including micro ribonucleic acid that can be transferred to target cells; and they can externalize phosphatidylserine that can bind annexin $\mathrm{V}$, forming a prothrombotic complex. Further, MPs can expose tissue factor, initiating thrombin generation and potential platelet activation. By exposing phosphatidylserine and tissue factor, MPs provide a procoagulant potential. Endothelial MPs are present in atherosclerotic plaque, and in patients who have acute coronary syndromes.

Conversely, MPs are capable of protecting endothelial cells and expressing cytoprotective and anti-inflammatory factors such as protein C. Further, MPs have plasmingenerating capabilities and this confers on them an important role in maintaining vascular patency. Microparticles seem to participate in inflammation, thrombosis, and angiogenesis, depending on the stimulus triggering their formation, the sites of formation, and the pathologic context. To say that MPs are complex is a gross simplification. Recent data show the potential beneficial effects of MPs on endothelial integrity, including stimulation of vascular repair, 
control of cell death mechanisms, and cytoprotective activities supported by activated protein C. Summation of the available information on the effect of MPs suggests a complex ambivalent role for these substances that can promote or inhibit coagulation, inflammation, or angiogenesis.

\section{A TOUR DE FORCE OF MICROPARTICLE- RELATED EFFECTS ON THE ENDOTHELIUM}

The article by $\mathrm{Fu}$ and colleagues, ${ }^{12}$ in the current issue of the Journal, attempts to unravel the complex impact of MPs on endothelial function in patients undergoing valvular heart operations using cardiopulmonary bypass (CPB). These authors evaluated the effect of MPs on multiple components of endothelial function. Their study represents a tour de force in demonstrating the contributions of MPs to endothelial dysfunction in patients who have valvular heart disease and are undergoing cardiac operations. No ambivalence accompanies their results: MPs from these patients are associated with significant impairment of physiologic markers of endothelium-dependent vasodilation mediated by endothelial nitric oxide synthase. Using a broad panel of in vitro physiologic testing, they found multiple contributions to endothelial dysfunction in patients with valvular heart disease, immediately before operation, and to an even greater extent, after cardiac valvular surgery using CPB. The fact that MPs have this effect in patients with valvular heart disease suggests a possible role of MPs as biomarkers of endothelial dysfunction, and perhaps more importantly, offers the possibility of therapeutic interventions that may alleviate endothelial dysfunction both before and after cardiac operations.

The study by Fu and colleagues ${ }^{12}$ does have some gaps. Although the authors found elevation of MP levels in patients who had valvular heart disease, the origin of the MPs is unclear. Platelets are the most likely source of circulating MPs, especially in patients subjected to CPB; but other sources of MPs are likely as well. Does the source of the MPs matter? Identifying the source of MPs helps identify the surface receptors on MPs, and the cellspecific binding sites may be key in initiating some physiologic effects of MPs. Limitations in identifying various contributions to the surface characteristics of MPs are a function of their small size, and hamper accurate characterization of the source of MPs. From a physiologic standpoint, the exact source of MPs is probably less important than the effects of multisource MPs.

The preparation of MPs requires careful control. Steps taken to harvest MPs can alter results of analyses. ${ }^{13}$ Preanalytic steps, such as delay before centrifugation of samples containing MPs, agitation during transport of the tubes containing MPs, and the exact centrifugation protocol all can affect the variability of results when testing MP effects.
Unfortunately, almost all of the harmful or beneficial effects of MPs, both those that appear in previously published literature and those presented by $\mathrm{Fu}$ and colleagues, ${ }^{12}$ have been identified through in vitro studies, because of the difficulty in measuring in vivo functional effects of MPs. New modifications of flow cytometry, the mainstay of measurement of MPs, may provide more real-time meaningful data regarding the in vivo effects of MPs in various biologic processes.

Fu and his colleagues ${ }^{12}$ made a careful and robust effort to accurately quantify the effects of MPs on endothelial function in their patient population. They found that MPs impair endothelium-dependent vasodilation by inhibiting nitric oxide synthase activity. Despite this well done study, this effect is likely only one of many. In the past 5 years, the number of investigations into the role of MPs in a wide range of physiologic processes increased dramatically. More information about MPs almost certainly will be forthcoming, and will likely have a major impact on more than just endothelial dysfunction. I look forward to seeing what lies ahead.

\section{References}

1. Herring JM, McMichael MA, Smith SA. Microparticles in health and disease. J Vet Intern Med. 2013;27:1020-33.

2. Klinkner DB, Densmore JC, Kaul S, Noll L, Lim HJ, Weihrauch D, et al. Endothelium-derived microparticles inhibit human cardiac valve endothelial cell function. Shock. 2006;25:575-80.

3. Muralidharan-Chari V, Clancy JW, Sedgwick A, D'Souza-Schorey C. Microvesicles: mediators of extracellular communication during cancer progression. $J$ Cell Sci. 2010;123:1603-11.

4. Wang CC, Tseng CC, Hsiao CC, Chang HC, Chang LT, Fang WF, et al. Circulating endothelial-derived activated microparticle: a useful biomarker for predicting one-year mortality in patients with advanced non-small cell lung cancer. Biomed Res Int. 2014;2014:173401.

5. Sansone R, Stanske B, Keymel S, Schuler D, Horn P, Saeed D, et al. Macrovascular and microvascular function after implantation of left ventricular assist devices in end-stage heart failure: role of microparticles. J Heart Lung Transplant. 2015;34:921-32.

6. Nascimbene A, Hernandez R, George JK, Parker A, Bergeron AL, Pradhan S, et al. Association between cell-derived microparticles and adverse events in patients with nonpulsatile left ventricular assist devices. J Heart Lung Transplant. 2014;33:470-7

7. Windelov NA, Johansson PI, Sorensen AM, Perner A, Wanscher M, Larsen CF, et al. Low level of procoagulant platelet microparticles is associated with impaired coagulation and transfusion requirements in trauma patients. J Trauma Acute Care Surg. 2014;77:692-700.

8. Wei Y, Zhao L. Passive lung-targeted drug delivery systems via intravenous administration. Pharm Dev Technol. 2014;19:129-36.

9. Al Kindi H, Paul A, You Z, Nepotchatykh O, Schwertani A, Prakash S, et al. Sustained release of milrinone delivered via microparticles in a rodent model of myocardial infarction. J Thorac Cardiovasc Surg. 2014;148:2316-23.

10. Lovren F, Verma S. Evolving role of microparticles in the pathophysiology of endothelial dysfunction. Clin Chem. 2013;59:1166-74.

11. Dignat-George F, Boulanger CM. The many faces of endothelial microparticles. Arterioscler Thromb Vasc Biol. 2011;31:27-33.

12. Fu L, Hu X-X, Lin Z-B, Chang F-J, Ou Z-J, Wang Z-P, et al. Circulating microparticles from patients with valvular heart disease and cardiac surgery inhibit endothelium-dependent vasodilation. J Thorac Cardiovasc Surg. 2015;150: 666-72.

13. Lacroix R, Judicone C, Poncelet P, Robert S, Arnaud L, Sampol J, et al. Impact of pre-analytical parameters on the measurement of circulating microparticles: towards standardization of protocol. J Thromb Haemost. 2012;10: 437-46. 